\title{
Analysis of National CO2 Emission Performance Based on Agricultural Emission Indicator
}

\author{
Xuanyou Chen ${ }^{1 *}$ Yue Zhang ${ }^{1}$ Pengxu Chen $^{2}$ Anduo Wang ${ }^{3}$ \\ ${ }^{1}$ Nansha College Preparatory Academy, Fok Ying Tung High School, Guangzhou, Guangdong 510000, China \\ ${ }^{1}$ Chu Kochen Honors College, Zhejiang University, Hangzhou, Zhejiang 310058, China \\ ${ }^{2}$ Tianjin Yinghua International School, tianjin, 301700, China \\ ${ }^{3}$ Beijing New Talent Academy, Beijing, 101300, China \\ *Corresponding author. Email: 22dchen@ncpachina.org
}

\begin{abstract}
This study uses data from the Food and Agriculture Organization (FAO) of the United Nations to investigate the relationship between agricultural $\mathrm{CO} 2$ emission, production, and related socioeconomic factors. First, we gather and process emission and production data, and then organize data based on country, year, and the source of emission. Second, we derive an Agricultural Emission Indicator measuring countries' ability to making efficient use of CO2 emission in agricultural production, and weight the score by a country's production capacity and composition. Third, we analyze the correlation between our Agricultural Emission Indicator and several economics-related measurements in an attempt to generalize trends and connections among countries. The three most important findings are: one, a country tends to be affected more and score higher on the type of food taking up the highest share of overall production; two, the emission scores for crop products including cereal and rice are more variable than scores for animal products including egg, meat, and milk; three, the performance of countries might be associated with their GDP, geographic, location, population. The findings offer insight into the role of economic activities in influencing agricultural emissions. We also develop a shiny app to show the emission and rank of a certain country and choose several typical countries for further analysis. However, the results of this study are limited by missing data, data inaccuracy, and a limited scope of investigation focusing only on $\mathrm{CO} 2$ and agricultural emissions. Future studies could consider expanding upon the scope of this research to include more GHG types and more economic activities, as well as gathering primary data from more reliable sources.
\end{abstract}

Keywords: Food and Agriculture Organization; Agriculture CO2 Emission; Socioeconomic Factors; Agricultural Emission Indicator

\section{INTRODUCTION}

The increasing prominence of environmental issues has been demanding environmental effort for years and recently become a topic of heated debate. On one side sits environmental scientists arguing for the need for the global economy to slow down the pace of development before human activities impose irreversible damage to the environment. On the other side are climate skeptics dismissing the existence of climate change and maintaining that to cut emissions is to stifle the growth of human society. How to walk a fine line between economic progress and environmental sustainability, therefore, becomes a critical issue to be discussed. Yet, no matter how the debate goes, the harsh reality of climate change calls for immediate action. Carbon dioxide (CO2), accounting for $55 \%$ of all greenhouse gas (GHG) emissions, is currently at its peak and projected to grow at an annual rate of $0.6 \%$ until $2050[1,2]$. Without further commitment to combating climate change, the average annual increase in temperature is projected to raise the global median by 2.6 to 3.1 degrees Celsius by 2100 [3].

The sources of human-driven global GHG emissions can be broken down into four major categories based on economic sectors: energy; agriculture, forestry, and land use; industry; and waste. This report will main focus on the agriculture sector to explore the relationship between agricultural production and emissions. Agriculture has become the second-largest source of GHG emissions, 
accounting for $18.4 \%$ of 2020 global GHG emissions [4]. The indispensable role of agriculture has arrested attention from scholars worldwide in its relation with society. Long and Tang (2021) commented that a "cointegration relationship" exists between economic growth and agricultural emission intensity. The role of agriculture also appears to vary among countries, although the over pattern shows a positive correlation between income and emissions [5]. The causality between the growth of income and GHG emissions three different directions in North America and Western Europe; Central and South America, Oceania and Japan; Asia and Africa [6]. Clearly, agricultural productions have played a pivotal role in determining the extent at which humanity depends and affects the environment. Therefore, it is crucial that the association between food production and GHG emissions be closely examined, and countries be assessed based on their performance in managing agricultural emissions to guide future policies. Numerous prior studies have undertaken to evaluate the intercorrelation between economic activities and GHG emissions. Tubiello et al. (2013) compiled global emission data from the Food and Agriculture Organization (FAO) covering information on production, economic indicators, commercials, and food balance in an effort to model trends in agricultural emissions over 1961 to 2010 and compare FAO data with other existing datasets [7]. Despite discovering that FAO estimates lower GHG emissions for certain emission measurements in comparison to data from the Environmental Protection Agency (EPA) and the Emissions Database for Global Atmospheric Research (EDGAR), the study observed that agricultural emissions total has been increasing steadily over the study period while the ratio of agricultural emissions to fossil fuel emissions continued to decline.

More recently, Yale Center for Environmental Law \& Policy published the 2020 Environmental Performance Index report, in which the environmental sustainability of 180 nations are assessed and ranked by 32 performance indicators among 11 issue categories. In particular, the climate change category models the trends and relationships among GHG emissions and GDP growth. The analysis indicates a close correlation between economic growth and stresses the importance of reducing the overall emissions rather than slowing down the rate of emissions relative to economic growth. For example, it has been found that emission intensity, calculated as emissions divided by GDP, sometimes goes onto the contrary direction to the total emissions when economic growth outperforms emission growth [8].

The focus of this study is to investigate the association between agricultural emissions and production. Specifically, we created an "Agricultural Emission Indicator" that assesses countries efficiency in utilizing $\mathrm{CO} 2$ emissions to maximize agricultural output weighted by the composition and capacity of food production. In the following three parts we will first, give an overview of our primary data source, the FAO database, describe the process of data treatment, and elaborate on the calculation of the Agricultural Emission Indicator; second, present and discuss associations and trends observed on the indicator; third, summarize our findings, limitations, and implications for future research.

\section{DATA}

\subsection{FAO data}

The primary data of this study comes from Food and Agriculture Organization http://www.fao.org/home/en/. Born in 1945, FAO works in over 130 countries worldwide with 194-member states, dedicating to the promotion of global food security. The official FAO website provides a wealth of data revolving environmental, agricultural, economic, and sustainability issues. The main datasets we use include Emission and Macro-Economic Indicators from the FAO website.

- Emission Tools

The dataset includes 7488 observations on 262 regions, from which 201 countries are selected for this research. The three types of observation are Emission intensity, $\mathrm{CO} 2$ Emissions and the Production of 14 food items, which are later generalized into cereal, rice, meat, milk, eggs.

\section{- $\quad$ Micro Indicators}

We use this dataset to learn about the Macroeconomic situation of different countries. We subset the dataset and only include 16550 observations on 201 countries' GDP, GDP-per-capita, GDP annual growth rate, GDP-percapita annual growth rate over 2008 to 2017.

\subsection{The Calculation of Agricultural Emission Indicator}

Countries throughout the world vary greatly in terms of the size of economy and the resources available for food production. Therefore, to make fair comparisons among countries, the production capacity of different types of food must be considered. For our own research purpose, we created a new data variable: the Agricultural Emission Indicator, referred in this report sometimes as the Weighted Emission Score. The indicator is defined as a score that assesses a country's ability to maximize agricultural production from $\mathrm{CO} 2$ emissions, and only $\mathrm{CO} 2$ emissions, relative to the world's performance, weighted by the quantity and composition of food production.

The primary data set used to calculate the score, the FAO emission intensity data, provides data of $\mathrm{CO} 2$ emissions volume in gigagrams, food production in tons, and emission intensity in $\mathrm{kg} \mathrm{CO} 2 / \mathrm{kg}$ production for 201 countries over 2008 to 2017 . The sources of emissions are 
divided into 14 types of food items in the primary data, which are categorized into five types of food in the treated data for the simplicity of calculation. The five types of food which are 1) Cereal, 2) Egg, 3) Meat, 4) Milk, and $5)$ Rice, each representing one or more sub-types. The process of calculating the score is described as follow:

First, we compute the aggregate emission intensity for every type of food, every country, and every year using Equation 1 . The intensity directly given by the data set is not adopted considering the difference in the categorization of food types. Countries with higher emission intensity generate more emissions per unit of food production. The emission intensity " $\mathrm{X}$ " of one food type consisting of " $\mathrm{N}$ " items is computed with the formula shown below:

$$
X=\frac{\sum_{i=1}^{N} \text { emission }_{i}}{\sum_{i=1}^{N} \operatorname{production}_{i}}
$$

Equation 1 for Emission Intensity

Second, we take the negative log of every single emission intensity value through Equation 2.

$$
\mathrm{X}=-\log \text { (intensity) }
$$

Equation 2 for Negative Logged Emission Intensity

Third, we transform the logged intensity into emission scores ranged from 0 to 100 for each food type separately using Equation 3. The transformation from an intensity value " $X$ " to a score " $Y$ " is accomplished through the following formula, where " $\max (\mathrm{X})$ " is the maximum value of the targeted food emission intensity out of all countries and all years, and "quantile $(\mathrm{X}, 0.05)$ " is the $5 \%$ quantile of the targeted food emission intensity out of all countries and all years:

$$
Y=\frac{X-\text { quantile }(X, 0.05)}{\max (X)-\text { quantile }(X, 0.05)}
$$

Equation 3 for Weighted Emission Intensity

NOTE that Equation 3 would possibly result in negative values, which were all manually converted into zeros.

Fourth, we multiply each score by the proportion of total agricultural production taken up by the corresponding type of food to produce the Weighted Emission Score, or the Agricultural Emission Indicator, aiming to assign more weight on the type of food a country specializes in producing with Equation 4. A higher score indicates a superior performance on maximizing agricultural production from $\mathrm{CO} 2$ emissions. The calculation of the Weighted Emission Score "W" involving " $\mathrm{N}$ " types of food employs the following formula, where the "score" is the emission score for the targeted food type and the "share" is the proportion of production taken up by the targeted food type:

$$
\mathrm{W}=\sum_{\mathrm{i}=1}^{\mathrm{N}} \text { score }_{\mathrm{i}} \times \text { share }_{\mathrm{i}}
$$

Equation 4 for Weighted Emission Score

\subsection{Data Processing}

After processing the above data sets separately, we constructed a new data frame for further explorations and analysis. The data frame contains a total of 2010 observations on 201 countries over 10 years from 2008 to 2017. There are 31 variables, which can be grouped into four main categories: production totals, emission totals, emission scores, GDP's.

\section{RESULTS}

The Weighted Emission Score over 10 years is modeled with a line graph for each of the 201 countries involved in this study along with the sub-scores of all the food types each country produces. The aim is to explore the trends and relationships among the Weighted Emission Score and the composition of food production. In the following sub-section, we give four examples to help understand our plots.

\subsection{Shiny App}

We develop a shiny depicting the performance of a selected country overtime. As shown in Figure 1, in the sidebar, we can choose the country that we want to study. And the plot down below can reflect the fluctuations in the emission score. Each plot is made for one country over 10 years. Each color represents one of the six scores. The $\mathrm{x}$-axis represents years, the $\mathrm{y}$-axis represents the score value, and the thickness of the lines represents the share of production. The rank of the country's score, with the highest score ranked the first, is shown underneath the graph. 


Select a country:
Afghanistan
Afghanistan
Albania
Algeria
Angola
Antigua and Barbuda
Argentina
Armenia
A...ntralin

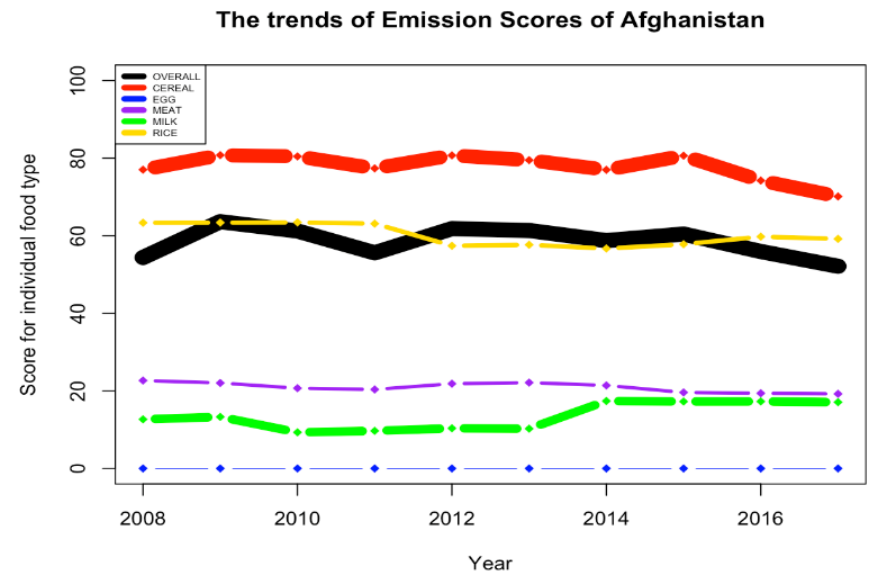

( ${ }^{*}$ The thickness of the lines represents the share of production) 10-Year Mean Ranking: 68 2017 Ranking: 107

Figure 1 Shiny App Demonstration

\subsection{Data Reliability}

Before any further analysis, a brief discussion of the sources and reliability of data is necessary to avoid misunderstandings. Data from the primary data base FAO in this research come from 7 types of sources: 1) official data; 2) unofficial figure; 3) FAO estimate; 4) calculated data 5) aggregate of official, semi-official, estimated, or calculated data; 6) FAO data based on imputation methodology 7) international reliable sources. Variance among sources would potentially lead to biased or inconsistent results, especially when data is provided by unofficial sources or small countries. In order to account for the reliability of data, we use the methodology introduced in the followings.

The first country we noticed with highly unstable emission score is Kuwait. Kuwait's cereal and rice emission scores are fluctuating abnormally. The scores on egg emissions demonstrate a similar level of volatility. Without discovering any occurrences of significant incidences taking place in Kuwait, this huge fluctuation could hardly be explained by a matter of probability. We therefore attribute this fluctuation in egg and cereal score to data problems, which would be further supported by an examination of the standard deviation, or the degree of variance among data, of each score.

Notice that the standard deviation for Kuwait caused by data problems is extremely high compared to normal cases. We then applied this method to all countries in an attempt to differentiate between data with normal variance and data with abnormal variance. We calculate five standard deviations, each representing the variance among 10-year scores of one type of food. Then we find that most of the countries demonstrate an acceptable level of standard deviation, with some outlier countries bearing higher risk of data problem. But without double checking with reliable sources for data accuracy the score could only serve as a reference, so there is no definite answer to whether a score value in this study is an accurate reflection of real-world performance or a misleading figure.

\subsection{Common Trend}

In this section we discuss some trends of the Weighted Emission Score that might imply some real-world implications.

Firstly, the Weighted Emission Score of a country tends to be affected by the sub-score of the type of food taking up the highest share of production. For example, despite Spain's egg meat, milk, and rice scores have remained relatively stable over years, the overall score does not move in accordance to the stationary pattern of but instead fluctuate with the score of cereal, which constitutes the majority of Spain's production. This observation should make mathematical sense as the calculation of the Weighted Emission Score has assigned food with more production more weight. In other words, the type of food a country specializes in producing holds more power in determining the agriculturalenvironmental performance of the country.

Secondly, comparisons among the position of subscores indicate that countries are more likely to score higher on the type of food weighted the most in production. According to the thickest line in the image, the score for cereal production, stays at a much higher position than the Weighted Emission Score. Supporting this trend, we found a 10-year average of 127 out of 201 countries to have a sub-score on the major food type greater than the overall Weighted Emission Score. One possible explanation would be that as the scale of 
production increases, a country would be able to make more efficient use of its inputs and develop techniques to boost production, or, as economists call it, achieve economies of scale. The increase in efficiency would allow a country to produce with lower emissions and thus receive a higher score on the corresponding standard. Nevertheless, it should never be taken for granted that some underlying mathematical mechanisms not recognized by this study have not come into place in creating this trend.

Further, we put countries into four groups based on the trajectory of emissions and production in order. The four trends are defined down below. Trend 1: Total
Emission increases; Total Production decreases; Trend 2: Total Emission increases; Total Production increases; Trend 3: Total Emission decreases; Total Production decreases; Trend 4: Total Emission decreases; Total Production increases.

To ascertain the association between the total production and total emission of countries, we create a matrix as shown in Table 1, in which the rows identify whether a country's Weighted Emission Score has increased at 2017 compared to 2008 and the columns identify the trend a country's emissions and production follow.

Table 1 Score Pattern against Trend

\begin{tabular}{|llllllll|}
\hline Trend1 & Trend2 & Trend3 & Trend4 & Row Sum & \\
\hline Overall Score Up & 2 & 62 & 13 & 32 & 109 \\
\hline Overall Score Down & 21 & 47 & 19 & 3 & 90 \\
\hline Column Sum & 23 & 109 & 32 & 35 & 199 \\
\hline Score Up & 9 & 57 & 41 & 91 & 55 \\
\hline
\end{tabular}

The results of trend1 and trend4 are easily comprehensible. Countries that generate fewer emissions and produce more agricultural product in absolute terms are doing well in maximizing production out of $\mathrm{CO} 2$ emissions and thus should receive score higher on our indicator. A total of 5 countries in trend 1 and trend 4 produce results that do not agree with the overall pattern. They are Libya, Portugal, Canada, Colombia, Ukraine. This inconsistency could possibly be attributed to the extent at which the overall score changes.

As shown by the example of Ukraine, the Weighted Emission Score has declined very slightly comparative to the 2008 level and therefore does not necessarily reflect a worsened performance. It could also be the case that the decline in the score on the major food type outweighs the increase in scores on other food types. In addition, as mentioned in the discussion of data reliability, some data from the primary data base FAO are not entirely credible and therefore may give rise to discrepancy among countries' actual performance and the performance measured by the indicator.

More interestingly, the results for trend 2 and trend 3 appear to be split more evenly. Both have about $40 \%$ to $60 \%$ of countries experiencing a score increase or decrease. Still, there is a big gap between the percentage of countries seeing a score uptick, with 49 more countries in trend 2 receiving an increase than in trend3. The superior performance of trend2, countries, whose emissions and production are both increasing could partially be attributed to the fact that growth in agricultural emissions and production is positively correlated to GDP growth. Thus, the increase in production and emissions might have implied a faster growing economy that provides more demands and opportunities for the agricultural sector to improve production.

This finding is supported by an overview of the regional distribution and GDP-per-Capita. Notice that the GDP-per-Capita and rate of growth is very different among groups. Trend2, where production and emissions are both growing, seem to be the third world countries whose economies are currently developing at a rapid pace. In contrast, countries in trend3, whose agricultural production and emissions both grow, are composed of mainly developed countries, presumably because these nations are able to shift the dependence from primary production onto secondary and tertiary production that involve more manufacturing and commercial services. Putting the 4 trends into group of 2, trend 1 and trend 3 have higher GDP-per-Cap, lower growth rate, and lower percentage of countries experiencing score increase, whereas trend 4 and trend 4 demonstrate the opposite, having lower GDP-per-Cap, faster growth rate and higher percentage of countries increasing in score.

Also, worth mentioning is the regional variance among the four groups. In the first two trends African and 
Asian countries are the most commonly seen, whereas in the latter two European and American countries have the highest frequency. This could possibly be linked to differences in the geography, resource availability, and environmental attitude among continents.

Lastly, the exploration of data reliability led to a surprising discovery that the standard deviation of cereal and rice emission score is significantly greater than that of egg, meat, and milk.

Note the quantity of production is accounted for in the process of calculation and thus a larger standard deviation is a direct indication of unstable performance. This observation could possibly be explained by the differing structure of farming and husbandry. The former is heavily dependent on the surrounding environment.

\subsection{Top and Bottom Ranked Countries}

We selected 20 countries whose mean rank over 10 years is either the top 10 or the bottom 10 and identify certain shared characteristics among top performers and bottom performers in this section. Table 2 shows a list of the top and bottom 10 countries.

Table 2 Top- and Bottom-Performing Countries

Top:

$\begin{array}{lc}\text { [1] "China, Hong Kong SAR" } & \text { "Democratic People's Republic of Korea" } \\ \text { [3] "France" } & \text { "Israel" } \\ \text { [5] "Japan" } & \text { "Lao People's Democratic Republic" } \\ \text { [7] "Latvia" } & \text { "Liberia" } \\ \text { [9] "Singapore" } & \text { "Togo" } \\ \text { Bottom: } & \\ \text { [1] "British Virgin Islands" } & \text { "Cook Islands" } \\ \text { [3] "Djibouti" } & \text { "Equatorial Guinea" } \\ \text { [5] "Maldives" } & \text { "Micronesia (Federated States of)" } \\ \text { [7] "Saint Kitts and Nevis" } & \text { "Samoa" } \\ \text { [9] "Tuvalu" } & \text { "Vanuatu" }\end{array}$

\section{- Population}

The size of top-performing countries is significantly greater compared to bottom-performing countries. The most populated country in the former group, Equatorial Guinea, is 680 hundred thousand behind in population compared to Latvia's 1,942 hundred thousand, which is the least population among the top-performing countries [9]. This observation could possibly be explained as the lack of efficiency in small scale production, as well as the relatively low availability of resources and technology in smaller countries.

\section{- GDP}

The top-performing countries we notice a more stable GDP growth rate, whereas the bottom-performing countries generally have less consistent growth rate over the years [10]. Most of the countries demonstrate a decline in the GDP growth rate in the period of recession, but the degree at which the rate slows down is far greater for bottom-performing countries. Economic lag and instability could serve as a contributing factor to the emission performance of bottom performers.

\section{- Continent}

Lastly, a brief overview of the continental distribution of top- and bottom- performers reveal some interesting trends. The result shows that countries that have higher overall emission scores which is the top 10 countries are all located in the northern and eastern hemisphere, while 7 out of 10 in the bottom performing group are located in the southern hemisphere. From a geographical perspective, one factor that might have contributed to the countries' performance is the area and location of agricultural land. The northern hemisphere contains $39.3 \%$ of land, $20.1 \%$ more than the $19.1 \%$ in the southern hemisphere. The mild weather in the north may also assist farmers to make more productive use of their input, which includes carbon emissions [11].

\section{CONCLUSION}

Overall, we have created the Agricultural Emission Indicator, in order to measure countries' performance on maximizing agricultural production and limiting $\mathrm{CO} 2$ emissions based on the capacity and composition of food production. Investigation of the score's association with a range of economic, geographic, and social variables lead us to discover that a country's major food production bears more determining power of the country's overall score, and tends to score higher than other sub-scores. Moreover, countries in different score range vary in terms of GDP growth, population, and geographical location: faster, more stable GDP growth and a location in the Southern hemisphere might be linked to a superior performance measured by the Agricultural Emission 
Indicator. The results are limited by first, the unavailability of data, as a result of which the scores for some countries or some years are missing Second, some primary data comes from unofficial sources, leading to a lack of data credibility. Third, the area of focus of this study is limited to $\mathrm{CO} 2$ and agriculture, and so our results is not directly applicable to other GHG emissions and economic sectors. Future research could consider including more elements in the calculation of the emission score to enhance the applicability of findings. The primary data should also be gathered from more reliable sources to avoid inaccurate measurements.

\section{REFERENCES}

[1] US Energy Information Administration. (2021, February 16). Energy and the environment explained. Retrieved July 31, 2021, from https://www.eia.gov/energyexplained/energyand-the-environment/outlook-for-futureemissions.php

[2] United States Environmental Protection Agency. (n.d.). Global greenhouse gas emissions data. Retrieved July 31, 2021, from https://www.epa.gov/ghgemissions/globalgreenhouse-gas-emissions-data

[3] Rogelj, J., Den elzen, M., Höhne, N., Fransen, T., Fekete, H., Winkler, H., Schaeffer, R., Sha, F., Riahi, K., \& Meinshausen, M. (2016). Paris agreement climate proposals need a boost to keep warming well below $2^{\circ} \mathrm{C}$. Nature, 534(7609), 631639.

[4] Ritchie, H., \& Roser, M. Our World in Data. (2020). Emission by sector. Retrieved August 4, 2021, from https://ourworldindata.org/emissions-bysector

[5] Long, D. J., \& Tang, L. (2021). The impact of socioeconomic institutional change on agricultural carbon dioxide emission reduction in china. PLOS One, 16(5).

[6] Coondoo, D., \& Dinda, S. (2002). Causality between income and emission: A country group-specific econometric analysis. Ecological Economics, 40(3), 351-367.

[7] Tubiello, F. N., Salvatore, M., Rossi, S., Ferrara, A., Fitton, N., \& Smith, P. (2013). The FAOSTAT database of greenhouse gas emissions from agriculture. Environmental Research Letters, 8(1).

[8] Wendling, Z. A., Emerson, J. W., de Sherbinin, A., Esty, D. C., et al. (2020). 2020 Environmental Performance Index. New Haven, CT: Yale Center for Environmental Law \& Policy. epi.yale.edu
[9] World Bank. (n.d.). Population, total. Retrieved August 8, 2021, from https://data.worldbank.org/

[10] World Bank. (n.d.). GDP (current US\$). Retrieved August 8, 2021, from https://data.worldbank.org/

[11] Briney, A. ThoughtCo. (2019, March 5). Geography of the northern hemisphere. Retrieved August 8, 2021, from https://www.thoughtco.com/geographyof-the-northern-hemisphere-1435555 